\title{
Versorgung von Traumapatienten mit dem „REBEL“-Set
}

Maximilian Kippnich, Yvonne Jelting, Martin Kraus, Thorsten Artmann, Thomas Wurmb, Peter Kranke

\begin{abstract}
Die zunehmende Terrorbedrohung und ein verändertes Einsatzspektrum für den Rettungsdienst erfordern eine Anpassung und Erweiterung von Notfallrettungsmitteln und Einsatztaktik. Ein Beispiel hierfür ist die bayerische „Handlungsempfehlung für Rettungsdienst bei besonderen Einsatzlagen (REBEL)“. Es werden 3 Fälle vorgestellt, bei denen „neue Hilfsmittel“ wie Hämostyptika, Thoraxverschlusspflaster und Tourniquet aus dem REBEL-Set bei Rettungsdiensteinsätzen angewandt wurden.
\end{abstract}

Trauma ist die häufigste Todesursache bei Personen bis 44 Jahre sowie die dritthäufigste Todesursache in allen Altersklassen in den westlichen Industrienationen [1]. Etwa 30-40\% der Schwerverletzten versterben an einer schweren Hämorrhagie - bis zu 50\% davon bereits präklinisch, insbesondere nach penetrierenden Traumata. Potenziell reversible Ursachen des traumatischen Herz-KreislaufStillstandes sind [2,3]:

- Hypovolämie

- Hämorrhagie

- Spannungspneumothorax

- Hypoxie, z. B. durch Atemwegsverlegung

- Perikardtamponade

Auch wenn die Versorgung schwerer Traumata gerade im bodengebundenen Notarztdienst in Deutschland ein eher seltenes Notfallbild darstellt, ist die Polytraumaversorgung ein zentraler Bereich der präklinischen Notfallmedizin, mit einem hohen Maß an Bewusstsein und Motivation aller Beteiligten. Dies zeigt sich auch in den zahlreichen und in den letzten Jahren immer weiter verbreiteten, zertifizierten Trauma-Kurssystemen - Pre Hospital Trauma Life Support $\left(\mathrm{PHTLS}^{\circledR}\right)$, International Trauma Life Support $\left(\right.$ ITLS $\left.^{\circledR}\right)$ - sowie der konsekutiven, „traumazentrierten“ Verbreitung einer algorithmusbasierten Versorgung. Dabei stimmen die Empfehlungen des PHTLS im hohen Maße mit den Empfehlungen der S3-Leitlinie Polytrauma überein [4].

Die in den letzten Jahren in Deutschland zunehmende Anzahl an Schadenslagen mit teilweise terroristischem Hintergrund hat nicht nur eine einsatztaktische Veränderung erfordert, sondern auch eine Modifikation bzw. Erweiterung der Notfallausstattung. Bei derartigen Einsätzen steht vorwiegend das penetrierende Trauma im Fokus [5]. So wurde in Bayern durch die oberste Rettungsdienstbehörde - das Bayerische Staatsministerium des
Innern, für Bau und Verkehr - eine Handlungsempfehlung für Rettungsdiensteinsätze bei besonderen Einsatzlagen (REBEL) erarbeitet und landesweit die Ausstattung aller Notfallrettungsmittel erweitert. Die folgenden 3 Kasuistiken beschreiben die Anwendung dieser REBEL-Ausstattung bei zivilen Notfällen. Sie verdeutlichen, dass die Erweiterung der Ausstattung in Kombination mit der Fortbildung aller Beteiligten - insbesondere im Bereich der Blutungskontrolle - primär auch bei nicht terroristischen Einsätzen sinnvoll eingesetzt werden kann.

\section{Fall 1: Hämostyptika}

Auf einer Bundesautobahn fährt ein Lkw auf ein stehendes Streufahrzeug auf. Der Fahrer des Lkw ist durch Intrusion des Lkw-Fahrerhauses schwer zugänglich und eingeklemmt. Im „Primary Survey“ ist Folgendes festzustellen:

- kritische Blutung aus dem Gesichtsbereich

- partiell verlegte Atemwege bei vorhandener Spontanatmung

- zentralisierte Kreislaufverhältnisse

- initialer Glasgow Coma Scale (GCS) = 8

Noch im Lkw wird mit mehreren Helfern mittels manueller Kompression durch Kompressen sowie mit Hämostyptika beschichteter Verbandmittel aus dem REBEL-Set versucht, die massive Blutung einzudämmen. Unmittelbar nach der technischen Rettung wird der sich im hämorrhagischen Schock befindliche, ca. 50-jährige Patient videolaryngoskopisch intubiert. Außerdem werden bei anhaltender Kreislaufinstabilität nach Notfallnarkose und hochdosierter Katecholamingabe (bis zu $20 \mu \mathrm{g} / \mathrm{min}$ Noradrenalin), u.a. über einen bereits während der technischen Rettung inserierten i. o. Zugang am proximalen Humerus, bei extrem zentralisierten Kreislaufverhältnissen 2 großlumige Thoraxdrainagen und eine Beckenschlinge angelegt. Präklinisch werden insgesamt 


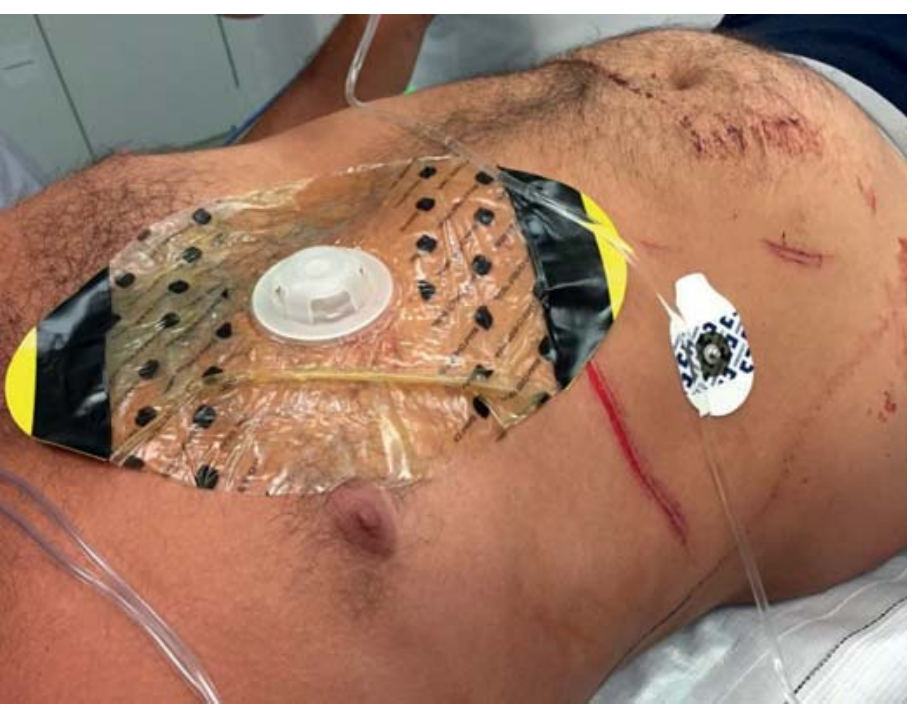

- Abb. 1 Thoraxverschlusspflaster mit Ventil an der ventralen Durchspießungswunde.

- 2 I Kristalloidlösung,

- $500 \mathrm{ml}$ Kolloidallösung sowie

- $1 \mathrm{~g}$ Tranexamsäure

als Kurzinfusion gegeben.

Die Traumaspirale (Ganzkörper-CT) im Schockraum zeigt neben einer mehrfragmentären, dislozierten Fraktur des Sinus frontalis und dislozierten Frakturen der rechten Orbita mehrere stabile Hals- und Brustwirbelkörperfrakturen sowie einen schmalen ventralen Pneumothorax links. Bei einem initialen $\mathrm{Hb}$ von $7,7 \mathrm{~g} / \mathrm{dl}$ und einer anzunehmenden Koagulopathie werden unmittelbar im Schockraum 2 Erythrozytenkonzentrate sowie $4 \mathrm{~g}$ Fibrinogen verabreicht.

Nach operativer Versorgung der Frakturen in mehreren Sitzungen und einem mehrwöchigen Klinikaufenthalt kann der Patient ohne Residuen in eine Rehabilitationseinrichtung entlassen werden.

\section{Fall 2: Thoraxverschlusspflaster}

Auf einer Baustelle verunfallt ein 32-jähriger Arbeiter: $\mathrm{Er}$ stürzt aus ca. 1,5 m Höhe auf einen Armierungsstahl und zieht sich dabei eine rechtsseitige thorakale Pfählungsverletzung zu. Die Stange penetriert den Thorax des Verunfallten von vorne kaudal der Mamille nach laterodorsal. Vor dem Eintreffen des Notarztes haben die Ersthelfer den Patienten bereits von der Metallstange gehoben, sodass der Bauarbeiter in Linksseitenlage ohne sichtbaren Fremdkörper am Unfallort aufgefunden wird. Die initiale Untersuchung ergibt:

- $\mathrm{GCS}=15$
- offensichtliche penetrierende Thoraxverletzung mit Ein- und Austrittswunde

- Schmerzen im rechten Handgelenk

- diverse Abschürfungen und Prellungen

Weitere relevante Verletzungen sind auch im Verlauf nicht feststellbar.

Der Patient zeigt Zeichen von Atemnot mit einer initialen peripheren $\mathrm{O}_{2}$-Sättigung von $92 \%$ ohne $\mathrm{O}_{2}$-Gabe und stärkste thorakale Schmerzen (numerische Ratingskala 10/10). Die Auskultation der Lunge zeigt keine Auffälligkeiten. Der erste systolische Blutdruck beträgt $100 \mathrm{mmHg}$, die Herzfrequenz 90/min. Es wird eine periphere Venenverweilkanüle am rechten Handrücken (18 G) angelegt und eine Analgosedierung durch die fraktionierte i.v.-Applikation folgender Substanzen durchgeführt:

- 0,4 mg Fentanyl

- $3 \mathrm{mg}$ Midazolam

- $2,5 \mathrm{~g}$ Metamizol

Weiterhin werden Thoraxverschlusspflaster mit Ventilmechanismus aus dem REBEL-Set auf die Penetrationswunden aufgebracht, um ein Eindringen von Luft in den Thorax zu verhindern ( $\bullet$ Abb. 1 ). Daraufhin wird der Patient mittels Schaufeltrage und Vakuummatratze in den Rettungswagen gebracht. Unter diesen Erstmaßnahmen verbessert sich der subjektive Zustand des Patienten deutlich.

Im Rettungswagen zeigen sich stabile Vitalparameter, der Patient gibt keine relevante Dyspnoe und erträgliche Schmerzen an. Daraufhin verzichtet man, die bereits vorbereitete Intubationsnarkose einzuleiten und eine präklinische Thoraxdrainage anzulegen. Der Transport in das nächstgelegene, überregionale Traumazentrum gestaltet sich problemlos.

Bei Aufnahme im Schockraum werden folgende Parameter dokumentiert:

- $\mathrm{GCS}=15$

- $\mathrm{O}_{2}$-Sättigung $=98 \%$ unter Gabe von $6 \mathrm{I} \mathrm{O}_{2} / \mathrm{min}$

- stabile Kreislaufparameter: Blutdruck 147/90 und Herzfrequenz 80/min

Das Ganzkörper-CT zeigt die Thoraxpenetration ohne Hinweis auf verletzte intrathorakale Organe. An der rechten Hand wird eine Spiralfraktur der Ossa metacarpalia II und III diagnostiziert, die später operativ versorgt wird. Unter intensivmedizinischer Überwachung wird im Verlauf noch die Therapie eines Pleuraergusses rechts mittels Thoraxdrainage erforderlich. Der Patient kann nach 2 Wochen aus dem Krankenhaus entlassen werden. 


\section{Fall 3: Tourniquet}

Ein 47-jähriger Motorradfahrer verunfallt durch eine frontale Kollision mit einem Lkw und wird teilweise von diesem überrollt. Beim Eintreffen des Notarztes zeigen sich:

- initialer GCS = 3

- massive Kreislaufinstabilität bei schwerem hämorrhagischem Schock

- traumatische Unterschenkelamputation links

- Femurfraktur links

- offene Wunden am Oberarm links

Als eine der ersten Maßnahmen wird ein Tourniquet aus dem REBEL-Set am linken Oberschenkel angelegt, um die kritische Blutung aus der Amputationsverletzung einzudämmen ( $\triangleright$ Abb. 2). Nach Einleitung einer Notfallnarkose erfolgt der unmittelbare luftgebundene Transport in den Schockraum eines überregionalen Traumazentrums.

Dort wird in der Ganzkörper-Computertomografie bei weiterhin instabilen Kreislaufverhältnissen - neben der offensichtlichen Amputationsverletzung - Folgendes festgestellt:

- anteriore Schultergelenksluxation links

- mehrfragmentäre Scapula- und distale Humerusfraktur links

- Rippenserienfraktur links (2. bis 9. Rippe)

- nicht dislozierte Fraktur des Os sacrum rechts

- mehrfragmentäre Fraktur des Acetabulums rechts

- obere und untere Schambeinastfraktur links mit Beteiligung der Symphyse

- nicht dislozierte intraartikuläre Fraktur der medialen Femurkondyle links

Bei einem initialen $\mathrm{Hb}$ von $8,5 \mathrm{mg} / \mathrm{dl}$ und der Notwendigkeit einer sofortigen operativen Versorgung der Verletzungen werden noch im Schockraum 4 Erythrozytenkonzentrate verabreicht.

Während der operativen Initialversorgung der führenden Verletzungen kann durch differenzierte Volumen-, Katecholamin- und Gerinnungstherapie eine zunehmende Kreislaufstabilisierung erreicht werden. Nach einem 7wöchigen Intensivaufenthalt und der operativen Versorgung der beschriebenen Verletzungen - sowie einigen Revisionseingriffen und der Nachresektion des Stumpfes - wird der Patient in stabilem Zustand in eine Rehabilitationsklinik verlegt.

\section{Diskussion}

Gerade bei schwerverletzten Patienten ist die zielgerichtete präklinische Versorgung für den weiteren Verlauf der Traumabehandlung von entscheidender Bedeutung. Buschmann et al. bezeichneten die Präklinik als einen der Brennpunkte des Traumamanagements [6]. Der

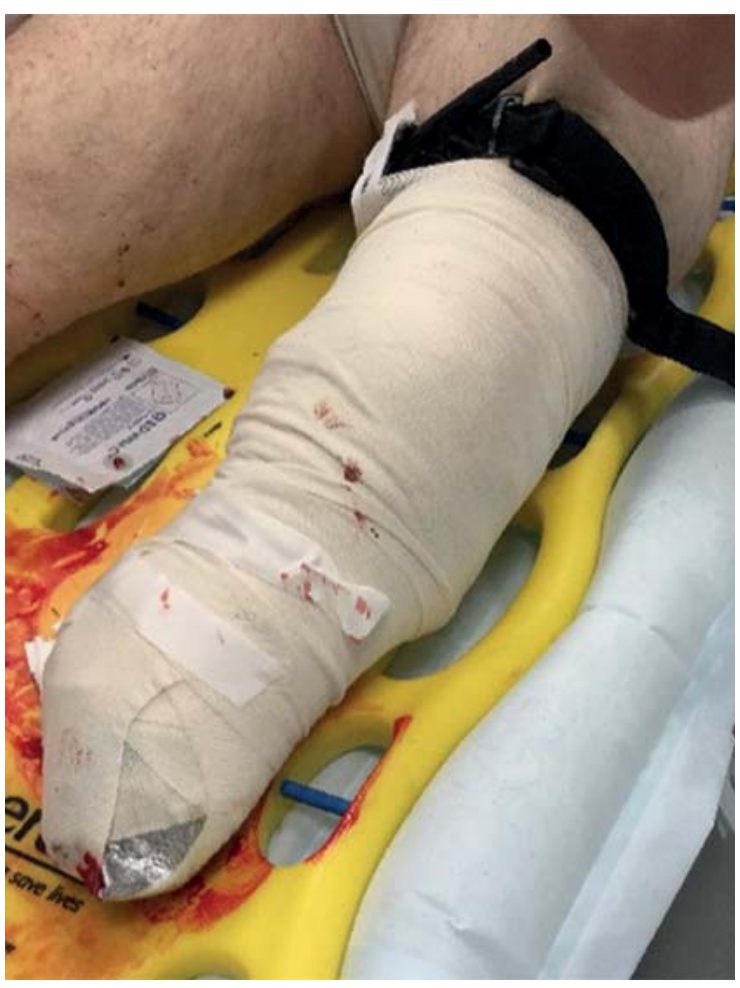

- Abb. 2 Tourniquet am linken Unterschenkel mit zusätzlich angebrachtem Druckverband.

schnelle Transport in das nächste geeignete Traumazentrum zur weiteren klinischen Versorgung - nach dem Konzept der „Golden Hour“ - ist weiterhin ein entscheidender Baustein [7]. Dennoch ist es unabdingbar, bei akut vital bedrohlichen Verletzungsmustern - trotz einer etwaigen Verlängerung der präklinischen Versorgungszeit - lebensrettende und teilweise invasive Maßnahmen zu ergreifen. Dazu zählen die Einleitung einer Notfallnarkose mit Atemwegssicherung und kontrollierter Beatmung bei drohender oder eingetretener Hypoxie oder die Entlastung eines Spannungspneumothorax [8-10].

\section{Frühzeitige Blutungskontrolle $<\mathrm{C}>$}

Die konsequente Blutungskontrolle bei starken bzw. potenziell lebensbedrohlichen Blutungen steht besonders im Fokus. Hier erfolgt eine Modifikation des klassischen $A B C D E-A l g o r i t h m u s ~ h i n ~ z u ~<C>A B C D E$, auch beim schweren Monotrauma [11]. Neben einer letalen Hämorrhagie kann durch die frühe gezielte Blutstillung eine konsekutive traumainduzierte Koagulopathie verhindert werden, die bei 30\% der Patienten bei Ankunft im Schockraum vorliegt [12]. Gegenüber rein supportiven Maßnahmen, wie z.B. der Volumentherapie, haben folgende Anwendungen eine deutlich höhere Priorität:

- frühzeitige Anlage einer Beckenschlinge bei entsprechender Unfallkinetik

- Blutstillung durch Anlage eines Tourniquets bei distalem Extremitätentrauma 
- Anwendung von Hämostyptika bei proximalen Blutungen bzw. am Körperstamm

Auch wenn die meisten Blutungen durch die Anlage eines konventionellen Druckverbandes kontrolliert werden können, ist es gerade bei penetrierenden Wunden entscheidend, die Wundhöhle mit geeigneten Verbandstoffen bzw. Hämostyptika in Form von Verbänden auszutamponieren und ausreichenden Druck auf die Wunde auszuüben [13]. Präklinisch sollen nur Hämostyptika in Form von Verbänden und nicht als Pulver verwendet werden [14].

\section{Antikoagulanzien}

Aktuell werden ca. 1\% der europäischen Bevölkerung mit Antikoagulanzien therapiert [15]. Hier kann es bereits durch Bagatelltraumata zu einer bedrohlichen Blutung kommen, die zielgerichtet therapiert werden muss. Gerade in Kombination mit einer größeren Wundfläche bietet sich die frühzeitige Anwendung eines Hämostyptikums auf der Basis von Chitosan an. Solche Hämostyptika sind durch ihre adhäsive bzw. mukoadhäsive Eigenschaften wirksam bei [16]:

- bestehender Koagulopathie

- Antikoagulation

- Hypothermie

Sie erreichen im Vergleich zu anderen Hämostyptika die schnellste und effektivste Blutstillung [17].

\section{Atemweg}

Nach Eindämmung der kritischen Blutung liegt im $<C>A B C D E-A l g o r i t h m u s$ der Fokus auf dem Atemweg. Dieser ist insbesondere auch bei Traumapatienten hinreichend in der S1-Leitlinie Notfallnarkose beleuchtet und soll nicht Bestandteil dieser Diskussion sein [18].

\section{Thoraxtrauma}

Ein weiteres Verletzungsmuster, das präklinisch häufig einer unmittelbaren Therapie bedarf, ist das Thoraxtrauma. Identifiziert wird dieses im Primary Survey bei der Untersuchung der Atmung: „B - Breathing“ (Dyspnoe, erhöhte Atemfrequenz/-arbeit, Schmerzen am Thorax, pathologische Auskultationsbefunde etc.). Konsekutiv können auch Probleme des Kreislaufs („C - Circulation“) entstehen, wie es z. B. beim Spannungspneumothorax der Fall ist. Es wird zunächst zwischen einem stumpfen und einem penetrierendem Thoraxtrauma unterschieden. Letzteres ist insbesondere bei besonderen Einsatzlagen wie Amoklauf oder Terror gehäuft zu beobachten (Schuss- bzw. Stichverletzungen). Auch eine Kombination aus stumpfem und penetrierendem Trauma ist denkbar (z. B. Explosion) [19].

Die initiale Therapie jeder respiratorischen Störung nach Trauma ist die hochdosierte $\mathrm{O}_{2}$-Gabe mittels High-FlowMaske [13].
Die wichtigsten Untersuchungsparameter bezüglich des Vorliegens eines relevanten Hämatopneumothorax bei Traumapatienten sind:

- thorakaler Schmerz

- Vorhandensein von Dyspnoe

- Auskultationsbefund mit einseitig abgeschwächten Atemgeräusch

Liegen bei einem Thoraxtrauma alle 3 Parameter vor, so liegt die statistische Wahrscheinlichkeit für einen relevanten Hämatopneumothorax > 99\%.

Bei Verdacht auf Pneumothorax muss der Patient engmaschig bezüglich Zeichen eines Spannungspneumothrorax untersucht werden. Neben dem einseitigen Fehlen des Atemgeräusches stehen hier vor allem zirkulatorische Symptome im Vordergrund. Der Spannungspneumothorax ist die häufigste reversible Ursache des traumatischen Herz-Kreislauf-Stillstands.

Besteht der Verdacht auf einen Spannungspneumothorax, muss die betroffene Pleurahöhle unmittelbar dekomprimiert werden. Dies kann zunächst mittels Thoraxentlastungspunktion mit einer großlumigen Kanüle erfolgen, bis personelle und materielle Ressourcen zur Verfügung stehen, um eine Thoraxdrainage zu legen. Alternativ kann auch eine Mini-Thorakotomie, z. B. in BülauPosition, durchgeführt werden, um eine akute Entlastung zu erwirken. Bei der Entlastungspunktion muss darauf geachtet werden, dass die Nadel eine ausreichende Länge von mind. $80 \mathrm{~mm}$ besitzt, um die Thoraxwand und die Pleura sicher zu perforieren [20,21]. Neben der MonaldiPosition im 2. bzw. 3. Interkostalraum medioklavikular ist auch die Bülau-Position im 4. bis 6. Interkostalraum der vorderen bzw. mittleren Axillarlinie möglich [21]. Dabei sollte man darauf achten, dass die Bülau-Drainage stets oberhalb der Mamillenlinie eingebracht wird, um einer Verletzung von benachbarten Organen (z. B. Leber) vorzubeugen.

Grundsätzlich ist der gesamte Thorax ventral und dorsal nach offenen Wunden zu untersuchen, auch wenn - wie in Fall 2 - die Wunden offensichtlich zu sein scheinen. Dies impliziert auch, den Patienten vollständig zu entkleiden. Beim Vorliegen offener Thoraxverletzungen sollten diese unmittelbar luftdicht verschlossen werden. Dadurch kann nicht nur eine subjektive Linderung der Atembeschwerden, sondern auch eine Verbesserung der Oxygenierung erzielt werden $[23,24]$. Zum Verschluss derartiger Wunden sind kommerziell hergestellte Thoraxverschlusspflaster die Mittel der Wahl, da durch diese ein adäquater luft- und blutdichter Verschluss erzielt werden kann. Verschlusspflaster mit Ventilmechanismus sollten bevorzugt werden, weil durch diese nicht nur im Thorax überschüssige Luft- und Flüssigkeitsmengen suffizient austreten können, sondern auch der Entwicklung eines Spannungspneumothorax vorgebeugt wird [25-27]. 
- Tab. 1 Inhalt des REBEL-Sets „Rettungsdienst“ und des REBEL-Sets „Bereitschaften“ in Bezug auf das ABCDE-Schema.

\begin{tabular}{|l|l|l|}
\hline ABCDE-Algorithmus & REBEL-Set Rettungsdienst & REBEL-Set Bereitschaften \\
\hline Airway (Atemweg) & & Wendl-Tuben \\
\hline Breathing (Beatmung) & $\begin{array}{l}\text { Thoraxpunktionsnadeln } \\
\text { Thoraxverschlusspflaster }\end{array}$ \\
\hline Circulation (Kreislauf) & $\begin{array}{l}\text { Tourniquet } \\
\text { Hämostyptika } \\
\text { Beckenschlinge }\end{array}$ & $\begin{array}{l}\text { Tourniquet } \\
\text { Notverband/Emergency Bandage }\end{array}$ \\
\hline Disability (neurologische Funktion) & $\begin{array}{l}\text { Verbandpäckchen } \\
\text { Z-folded Gauze }\end{array}$ \\
\hline $\begin{array}{l}\text { Exposure \& Environment (Entkleiden/ } \\
\text { Wärmeerhalt, Umweltbedingungen) }\end{array}$ & zusätzliche SAM-Splint-Schienen & $\begin{array}{l}\text { Wärmeschutzfolie } \\
\text { Patienten-Transfer-Unterlage/Umbettungstuch } \\
\text { Leuchtstift/Knicklicht }\end{array}$ \\
\hline
\end{tabular}

Sowohl nach Thoraxverschluss mittels Verschlusspflaster als auch nach Anlage einer Thoraxdrainage muss engmaschig evaluiert werden, ob die getroffenen Maßnahmen effizient sind. Dies gilt insbesondere nach Einleitung einer Notfallnarkose mit maschineller Beatmung. In diesem Zusammenhang sei nochmals auf die S1-Leitlinie Notfallnarkose verwiesen [18]. Auch wenn die aufgeführten Verletzungsmuster und das beschriebene Vorgehen insbesondere bei besonderen Einsatzlagen wie Amok oder Terror - erwartet werden, muss man mit derartigen Verletzungen auch bei zivilen Notfallbildern rechnen. Exemplarisch hierfür stehen die aufgeführten Fallbeispiele $1-3$.

\section{Rettungsdiensteinsätze bei besonderen Einsatzlagen (REBEL)}

Die notfallmedizinischen Aspekte bei „lebensbedrohlichen Einsatzlagen“ wurden nochmals in den jüngst veröffentlichten Ergebnissen eines nationalen Konsensusgesprächs zum Thema „Zusammenarbeit von Rettungskräften und Sicherheitsbehörden bei bedrohlichen Lagen“ betont [28]. Aufgrund der veränderten Bedrohungslage und der daraus resultierenden gestiegenen Anforderungen an den Rettungsdienst gab die oberste bayerische Rettungsdienstbehörde - das Bayerische Staatsministerium des Innern, für Bau und Verkehr - am 09.06.2016 „Handlungsempfehlungen für Rettungsdiensteinsätze bei besonderen Einsatzlagen/Terrorlagen (REBEL)“ heraus. Neben einer an derartige Lagen angepassten Einsatztaktik wurde auch eine Erweiterung der medizinischen Ausstattung der Notfallrettungsmittel empfohlen. Nicht nur der Rettungsdienst, sondern auch ehrenamtliche Einheiten des Katastrophenschutzes - z. B. die Bereitschaften des Bayerischen Roten Kreuzes - wurden zeitnah mit sog. REBEL-Sets ausgestattet [19]. In - Tab. 1 ist das REBEL-Set „Rettungsdienst“ und das REBEL-Set „Bereitschaften“ in Bezug auf das ABCDE-Schema dargestellt. Darin sind enthalten:

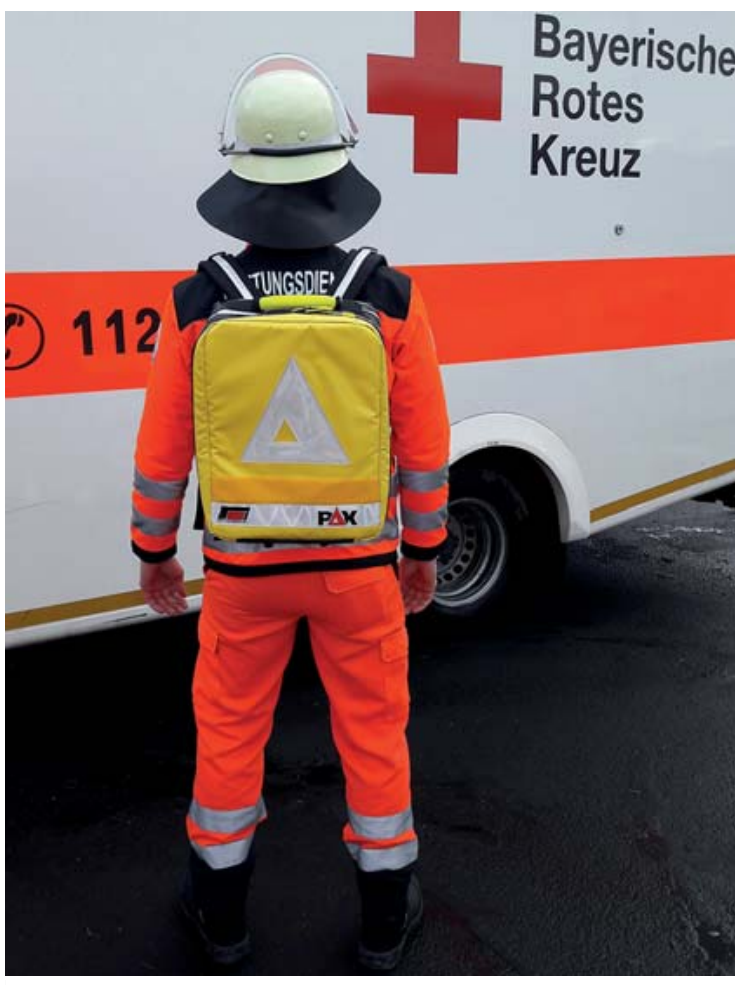

- Abb. 3 REBEL-Set „Rettungsdienst“ in einem Zusatzrucksack auf einem Rettungstransportwagen.

- zusätzliche Verbandsmaterialien

- Tourniquets

- Hämostyptika

- Thoraxpunktionsnadeln

- Thoraxverschlusspflaster

Außerdem beinhaltet die Empfehlung die Vorhaltung von Traumaequipment, welches aber bereits auf den Notfallrettungsmitteln in Bayern flächendeckend vorhanden ist (z. B. Beckenschlinge). In der Praxis ist je nach Rettungsdienstbereich diese Zusatzausstattung entweder in die 


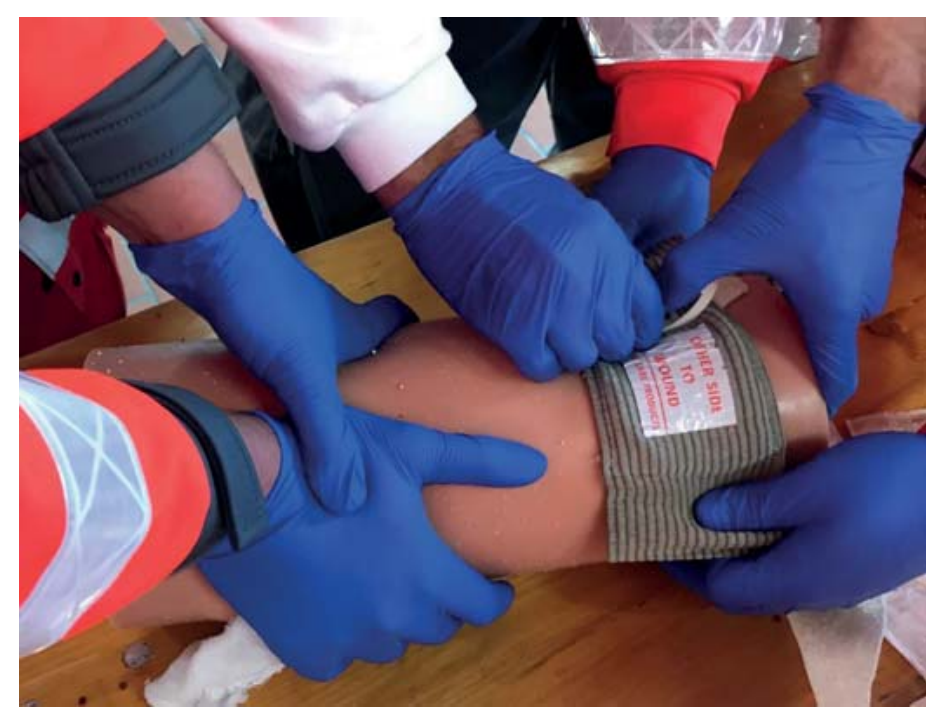

- Abb. 4 Praktische Übung zur Blutungskontrolle mittels spezieller Verbandmittel am Modell.

Standard-Notfallausrüstung integriert oder wird in einem gesonderten Rucksack mitgeführt ( $\bullet$ Abb. 3).

Die Bevorratung dieser neuen Devices bietet neue Möglichkeiten der zielgerichteten Versorgung akut vital bedrohlicher Zustände. Die stringente algorithmusbasierte Versorgung ( $\angle C>A B C D E)$ stellt die Grundlage hierfür dar, insbesondere auch für die Anwendung durch nicht ärztliches Rettungsdienstpersonal. Für eine sichere Anwendung dieser Devices ist eine fundierte Ausbildung aller an der Rettungskette beteiligten Helfer notwendig. Exemplarisch ist hier die „REBEL-Schulung“ für jeden Sanitäter der Bereitschaften des Bayerischen Roten Kreuzes zu nennen, bei der nach einer theoretischen Einführung die praktische Anwendung an Simulatoren und Modellen geübt wird ( $\bullet$ Abb. 4). Bestenfalls wird in Zukunft die Indikationsstellung und der Umgang mit den beschriebenen "neuen" Hilfsmitteln schon in die Notfallsanitäterund Notarztausbildung integriert.

Zusammenfassend sollte jeder im Rettungsdienst Tätige bei der Versorgung von Traumapatienten niederschwellig an die beschriebene Zusatzausstattung denken, vor dem tatsächlichen Einsatz die Indikation aber kritisch prüfen. Auch wenn diese im Rahmen der veränderten Bedrohungslage durch Terror angeschafft wurden, dürfte der Großteil des Einsatzgebietes in zivilen Notfallbildern liegen.

\section{KERNAUSSAGEN}

- Im Rahmen der veränderten Bedrohungslage durch Terror werden bundesweit die Notfallrettungsmittel mit Zusatzmaterial bestückt, das auf die zu erwartenden Verletzungsmuster abgestimmt ist.

- Im Freistaat Bayern haben Einheiten des Rettungsdienstes und Katastrophenschutzes das sog. REBEL-Set nachgerüstet (auf Basis der Handlungsempfehlung für Rettungsdiensteinsätze bei besonderen Einsatzlagen des Bayerischen Staatsministeriums des Innern, für Bau und Verkehr).

- Diese Zusatzausstattung umfasst z. B. Thoraxverschlusspflaster und -entlastungskanülen, Tourniquets und Hämostyptika sowie weitere spezielle Verbandmittel.

- Unabhängig von der Schadenslage (zivile Notfälle oder besondere Einsatzlagen) stellen Verbluten, Atemwegsverlegung und Spannungspneumothorax die häufigsten vermeidbaren Todesursachen von Traumapatienten dar.

- Durch eine stringente, algorithmusbasierte Notfalltherapie (z. B. <C>ABCDE-Algorithmus) können derartige Zustände zügig identifiziert und unter Einbeziehung der „neuen“ Hilfsmittel unmittelbar therapiert werden.

- Die kritische Indikationsstellung und die Anwendung dieser Hilfsmittel erfordert eine fundierte Schulung in Theorie und Praxis von ärztlichem und nicht ärztlichem Rettungsdienstpersonal.

- Derartige Schulungen sollten frühzeitig schon in die Ausbildung angehender Notfallsanitäter und Notärzte integriert werden.

Interessenkonflikt

Die Autoren geben an, dass kein Interessenkonflikt vorliegt.

\section{Autorinnen/Autoren}

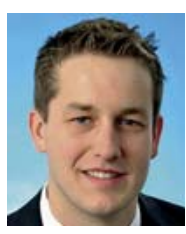

\section{Maximilian Kippnich}

Dr. med., Jahrgang 1990. 2009-2015 Studium der Humanmedizin in Würzburg. Seit 2015 Facharztausbildung Anästhesiologie und Intensivmedizin am Universitätsklinikum Würzburg (Sektion Notfall- und Katastrophenmedizin). Bereitschaftsarzt und Zugführer im Bayerischen Roten Kreuz, Lehrkraft an der Berufsfachschule für Notfallsanitäter in Würzburg. 


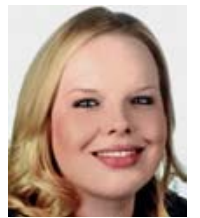

\section{Yvonne Jelting}

Jahrgang 1989. 2009-2015 Studium der Humanmedizin in Würzburg. Seit 2015 Prüfärztin und Assistenzärztin am Uniklinikum Würzburg. Klinik und Poliklinik für Anästhesiologie, Universitätsklinikum Würzburg.

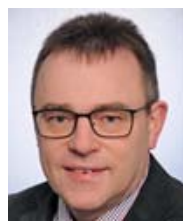

\section{Martin Kraus}

Dr. med., Jahrgang 1965, Facharzt für Anästhesie, Notfallmedizin, Ärztliches Qualitätsmanagement. Niedergelassener Anästhesist mit Tätigkeit in der Rotkreuzklinik Würzburg, ärztlicher Bezirksbeauftragter Rettungsdienst an der Regierung von Unterfranken, leitender Notarzt und Notarzt in der Land- und Luftrettung.

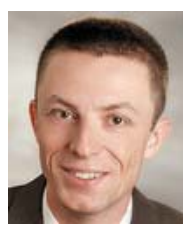

\section{Thorsten Artmann}

Jahrgang 1981. 2007-2013 Studium Humanmedizin in Würzburg, Assistenzarzt an der Klinik für Anästhesie und Intensivmedizin, Cnopfsche Kinderklinik/Klinik Hallerwiese, Nürnberg; Notarzt, Feuerwehrarzt Freiwillige Feuerwehr Würzburg-Lengfeld, Lehrkraft Berufsfachschule für Notfallsanitäter.

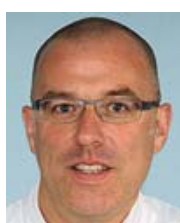

\section{Thomas Wurmb}

Univ.-Prof. Dr. med., Jahrgang 1969. Medizinstudium in Heidelberg. Seit 1997 an der Klinik und Poliklinik für Anästhesiologie Universitätsklinikum Würzburg. Seit 2015 Universitätsprofessor für Notfall- und Katastrophenmedizin. Vorstandsmitglied der Deutschen Arbeitsgemeinschaft Krankenhaus Einsatzplanung (DAKEP e.V.), Leiter der AG Trauma- und Schockraummanagement des Arbeitskreises Notfallmedizin der DGAI.

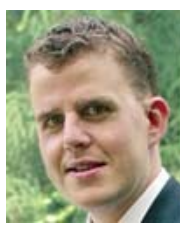

\section{Peter Kranke}

Univ.-Prof. Dr. med., MBA, Oberarzt, Leiter der klinischen Forschung und Lehrbeauftragter an der Klinik und Poliklinik für Anästhesiologie des Universitätsklinikums Würzburg. Klinisch verantwortlich für Anästhesie in der Gynäkologie und Geburtshilfe.

\section{Korrespondenzadresse}

Univ.-Prof. Dr. med. Peter Kranke, MBA

Klinik und Poliklinik für Anästhesiologie

Universitätsklinikum Würzburg

Oberdürrbacher Straße 6

97080 Würzburg

kranke_p@ukw.de

\section{Wissenschaftlich verantwortlich} gemäß Zertifizierungsbestimmungen

Wissenschaftlich verantwortlich gemäß Zertifizierungsbestimmungen für diesen Beitrag ist Univ.-Prof. Dr. Peter Kranke, Würzburg.

\section{Literatur}

[1] Pfeifer R, Tarkin IS, Rocos B et al. Patterns of mortality and causes of death in polytrauma patients - has anything changed? Injury 2009; 40: 907-911

[2] Kleber C, Giesecke MT, Tsokos M et al. Trauma-related preventable deaths in Berlin 2010: need to change prehospital management strategies and trauma management education. World J Surg 2013; 37: 1154-1161

[3] Kauvar DS, Lefering R, Wade CE. Impact of hemorrhage on trauma outcome: an overview of epidemiology, clinical presentations, and therapeutic considerations. J Trauma 2006; 60: $3-11$

[4] Häske D, Stuke L, Bernhard M et al. Comparison of the Prehospital Trauma Life Support recommendations and the German national guideline on treatment of patients with severe and multiple injuries. J Trauma Acute Care Surg 2016; 81: 388-393

[5] Henke TJ, Freund F, Wieprich D et al. Der Terroranschlag von Berlin - Die Vorgeschichte, der Einsatz und die Konsequenzen aus präklinischer Sicht. Notarzt 2017; 33: 54-60

[6] Buschmann C, Poloczek S, Giesecke MT et al. Vermeidbare Todesfälle nach Trauma - Epidemiologie, Todesursachen und Managementfehler. Notarzt 2013; 29: 91-108

[7] Fischer M, Kehrberger E, Marung $\mathrm{H}$ et al. Eckpunktepapier 2016 zur notfallmedizinischen Versorgung der Bevölkerung in der Prähospitalphase und in der Klinik. Notfall Rettungsmed 2016; 19: 387-395

[8] Kleber C, Giesecke MT, Tsokos M et al. Trauma-related preventable deaths in Berlin 2010: need to change prehospital management strategies and trauma management education. World J Surg 2013; 37: 1154-1161

[9] Hilbert-Carius P, Wurmb T, Lier H et al. Empfehlungen der S3Leitlinie Polytrauma/Schwerverletztenbehandlung 2016 für die Präklinik. Eine praxisorientierte Darstellung. Notfall Rettungsmed 2018; 21: 4-13

[10] Bernhard M, Helm M, Lechleuthner A et al. Erstversorgung vor Ort oder schnellstmöglicher Transportbeginn? Initial treatment on-scene or rapid transportation. Notfall Rettungsmed 2017; 20: 579-585

[11] Hodgetts T], Mahoney PF, Russell MQ et al. $A B C$ to $\angle C>A B C$ : redefining the military trauma paradigm. Emerg Med J 2006; 23 : 745-746

[12] Maegele M, Lefering R, Yucel $N$ et al. Early coagulopathy in multiple injuriy: an analysis from the from the German Trauma Registry on 8742 patients. Injury 2007; 38: 298-304

[13] Deutsche Gesellschaft für Unfallchirurgie. S3 - Leitlinie Polytrauma/Schwerverletzten-Behandlung (Juli 2016). Im Internet: http://www.awmf.org/uploads/tx_szleitlinien/012019l_ S3_Polytrauma_Schwerverletzten-Behandlung_2017-08.pdf; Stand: 11.06 .2018

[14] Josse F, Helm M, Kulla M et al. Präklinische Blutstillungsmaßnahmen: Hämostyptika. Notarzt 2015; 31: 153-157

[15] Cervera A, Amaro S, Chamorro A. Oral anticoagulant-associated intracerebral hemorrhage. J Neurol 2012; 259: 212-224

[16] Köksal O, Ozdemir F, Cam Etöz B et al. Hemostatic effect of a chitosan linear polymer (Celox ${ }^{\circledR}$ ) in a severe femoral artery bleeding rat model under hypothermia or warfarin therapy. Ulus Travma Acil Cerrahi Derg 2011; 17: 199-204

[17] Lier H, Bernhard M, Knapp J et al. Ansätze zur prähospitalen Gerinnungstherapie. Anaesthesist 2017; 66: 867-878

[18] Deutsche Gesellschaft für Anästhesiologie und Intensivmedizin (DGAI). Handlungsempfehlung zur prähospitalen Notfallnarkose beim Erwachsenen (März 2015). Im Internet: http:// 
www.awmf.org/uploads/tx_szleitlinien/001-030l_S1_Praehospitale_Notfallnarkose_Erwachsene_2015-03.pdf; Stand: 11.06.2018

[19] Sefrin P. Besondere Lage - Terroranschlag. Notarzt 2017; 33: 68-80

[20] Clemency BM, Tanski CT, Rosenberg M et al. Sufficient catheter length for pneumothorax needle decompression: a metaanalysis. Prehosp Disaster Med 2015; 30: 249-253

[21] Powers WF, Clancy TV, Adams A et al. Proper catheter selection for needle thoracostomy: A height and weight-based criteria. Injury 2014; 45: 107-111

[22] Laan DV, Vu TD, Thiels CA et al. Chest wall thickness and decompression failure: A systematic review and meta-analysis comparing anatomic locations in needle thoracostomy. Injury 2016; 47: 797-804

[23] Buttler FK, Dubose JJ, Otten EJ et al. Management of Open Pneumothorax in Tactical Combat Casualty Care: TCCC Guidelines Change 13-02. J Spec Oper Med 2013; 13:81-86

[24] Kotora JG, Henao J, Littlejohn LF et al. Vented chest seals for prevention of tension pneumothorax in a communicating pneumothorax. J Emerg Med 2013; 45: 686-694
[25] Arnaud F, Maudlin-Jeronimo E, Higgins A et al. Adherence evaluation of vented chest seals in a swine skin model. Injury 2016; 47: 2097-2104

[26] Kheirabadi BS, Terrazas IB, Miranda $\mathrm{N}$ et al. Do vented chest seals differ in efficacy? An experimental evaluation using a swine hemopneumothorax model. J Trauma Acute Care Surg 2017; 83: 182-189

[27] Kheirabadi BS, Terrazas IB, Koller A et al. Vented versus unvented chest seals for treatment of pneumothorax and prevention of tension pneumothorax in a swine model. J Trauma Acute Care Surg 2013; 75: 150-156

[28] Hossfeld B, Adams HA, Bohnen R et al. Zusammenarbeit von Rettungskräften und Sicherheitsbehörden bei bedrohlichen Lagen: Ergebnisse eines nationalen Konsensusgesprächs. Anästh Intensivmed 2017; 58: 573-583

\section{Bibliografie}

DOI https://doi.org/10.1055/s-0044-102122

Anästhesiol Intensivmed Notfallmed Schmerzther 2018; 53: 551-558 @ Georg Thieme Verlag KG Stuttgart · New York ISSN 0939-2661 